chial tubes, the cough becoming much less frequent. In the third case it was rejected by the stomach. Leeches were very serviceable to children, in whom the air pas. sages were much affected. The attacks, however, in children were seldom severe, and usualy required merely an aperient, followed by the administration of a few fever powders, the following formula for which I found very useful :-

Px Chloride of mercury, 3 grains; ipecacu. anha, 3 grains ; white sugar, 10 grains. Mix. Divide into six powders, of which one to be taken every third hour.

Leeches, applied to the temples, in all cases where the head was much affected, afforded relief.

Under these different modes of treatment most of the patients recovered speedily. I did not lose a single case, nor is one of the patients whom I treated for this complaint at present under medical care. I must also add, that those cases in which I had recourse to venesection, were of the shortest duration, and followed by the least debility, the inflammatory action in most cases terminating rapidly. I mention this fact, chiefly to show that the very general objection to blood-letting in this disease, on account, as it is stated of the debility which ensues, is unfounded, and calculated to prevent medical men from availing themselves of a remedy which would, I feel persuaded, in many cases have prevented its fatal termination, and also the dangerous and disagreeable sequele which have so frequently followed an expectant mode of practice. It is my firm conviction, that the early abstraction of a few ounces of blood will often check an inflammatory action, even of a specific nature, which, if allowed to run its course for twenty-four or forty-eight hours, would produce much greater debility than could arise from a moderate venesection.

About the commencement of February, I was requested to visit three patients attacked with violent hæmoptysis. This formidable disease was in each case consequent upon the influenza. On inquiry, $I$ found that no active treatment had been pursued. These hæmorthages were suppressed by the administration of the sulphate of alumina, and the patients recovered under the usual treatment. I believe these hæmorrhages might have been prevented, by a judicious use of the lancet.

In conclusion, I would not have it supposed that $I$ am an advocate for bleeding in every case of such an epidemic. I only wish to urge the necessity of examining closely into the nature, seat, extent, \&c. of the inflammatory action in such diseases, instead of prescribing according to their names. Were such an investigation always made, I think there are few medical men who would assert that bleeding is injurious in the influenza, or that either a stimulant or an expectant practice should be adopted. I remain, Sir, yours very respectfully, James Bedingfield Bryan, M.D. M.R.C.S.E., \&c.

Stowmarket, March 7, 1837 .

\section{THE INFLUENZA IN SHROPSHIRE.}

\section{To the Editor of The Lancet.}

SIR:-I have been actively engaged in attending cases of influenza; and the results of the treatment employed induce me to send to you this communication. Between 60 and 70 cases daily came under my immediate superintendence; and I investigated each case with all the accuracy that the opportunity afforded.

Persons attacked with this epidemic, I divided into three divisions :-1 st, Acute Peripneumony ; 2nd, Catarrh ; and 3dly, $\mathrm{Py}$ rexia.

Acute Peripneumony.-The symptoms were rigour and increased heat, and other symptoms of pyrexia. An acute fixed pain was felt in some part of the thorax, affecting the back and shoulders, with a considerable degree of difficulty in breathing, generally increased during inspiration; a violent dry cough, pain, and dulness in the head; the face, in some, oedematous, attended with turgescence and fushing of the face; great anxiety and restlessness; the pulse was, for the most part, frequent, full, strong, and hard, sometimes soft and weak; the tongue dry and red; intense thirst; hot skin; a high coloured state of the urine. The lancet, in these cases, I considered to be the archor of hope. I bled with freedom, in the first instance, and repeated the venesection, ac. cording to the urgency of symptoms. The blood drawn from the arm was cupped and buffy. In some cases I applied a blister to the sternum, and gave the following formula:-

R Poudered digitalis, and ipecacuania, of each 1 grain; nitrate of potash, 10 grains. Make a powder. Take one three times a day.

R Comp. powder of ipecac. 10 grains; antimonial pouder, 6 grains. Make a pow. der. Take one every night.

R Liquor of tart. of antimony, 1 drachm; spirit of nitrous ether, 2 draehms; dis. tilled water, 1 ounce. Make a draught. Take a fourth part every hour.

Aperient medicines and the pediluviam were ordered every night, and under this treatment the patient recovered,

The Caiarrh.-In others attacked with this disease, there were evident signs of inflammation of the mucous membrane lining the bronchia, and of the schneiderian, of mucous membrane of the nose; slight difficulty of breathing, with transitory pains about the chest; fulness of the nose; pain of 
the head, and secretion from the part; pyrexia; redness, and watering of the eyes; hoarseness and sore throat; cough, sometimes violent; tongue dry, and some thirst ; urine of a red colour. In these attacks I first promoted diaphoresis by the pediluvia, and mild dilnent drinks; the cough was mitigated by balsamic and mucilaginous mixtures; the bowels were kept regular by aperient medicines; when the patient complained of rawness in the wind pipe, I ordered inhalation of the steam of hot water, which $I$ also found to be exceedingly useful, in not only alleviating the pain of the head, but also the cough. Under this treatment patients got well.

Pyrexiu, with an eruption, appeared in some cases. The tongue was particularly red; there was slight disturbance of the sensorium. In these cases I gave emetics, purging medicines, and a fever mixture, and the patients all recovered.

The stethescopic signs in these diseases I generally observed to be resonance, pectoriloquy, and wegophony. I consider that there is a diffidence in employing energetic remedies in the early stage of the disease (epidemic catarrh) which has protracted it, and rendered its severity formidable and fatal, as, for instance, pneumonia, and those acute phlegmonous inflammations which occur in this disease. I think that there has been an unnecessary timidity, or, rather, a mistaken fear, as regards venesection.

I have the honour to be, Sir, your obedient humble servant,

$$
\text { William Weld Phillips. }
$$

Hales Owen, March 13th, 1837.

\section{INFLUENZA AND CHOLERA}

\section{COMPARED AND CONTRASTED.}

Tre obscurity in which these two affections are enveloped, renders desirable every attempt to elucidate their phenomena, and with this view the following reflections are offered. To draw comparisons, and to mark contrasts, form useful exercises to the philosopher, and as few diseases present so many striking analogies and distinctions, as influenza and cholera, some profit may be derived by placing them in such a position.

They are both of an epidemic nature, arising, passing over the face of the earth, and disappearing, in a mysterious manner. Both seem to be influenced by the season of the year, or by the state of the atmosphere as regards heat and moisture. The course of both is, mostly, from east to west, especially in this country, where the metropolis has, in general, been the first to suffer, and, subsequently, the more western parts.

Influenza passes rapidly over a consider- able tract of country; a great majority of all classes, both high and low, suffer from it; whereas the progress of cholera is more slow; the proportion of individuals attacked is less, and it rages chiefly amonust the lowest and most dissolute classes. The fatality of infuenza is slight; that of cholera is excessive; and in both affections a fatal result is mostly observed in the aged and afflicted. I have said, that influenza attacks all ránks in an equal degree; but if one class be more susceptible to its influence than another, it is the higher grades of society; whereas cholera, as I have remark: ed, prevails amongst the lowest classes. Cholera rarely attacks infants, but they are subject to influenza, and have it in a severe form, which often terminates fatally.

In both affections the mucous membrane exhibits the chief morbid appearances. In influenza, the mucous tissue lining the schneiderian cavities and bronchial tubes become the seat of inflammatory action. In cholera, that of the stomach and bowels is found in a highly congested state. Thus, we observe a similarity in the tissue affected, and a difference in the locality, and in the nature of the affection, characterising these two disorders. Impediment to the respiratory process attends influenza, and derangement of the digestive, marks cholera. In both, an unaccountable degree of depression and oppression is felt, the circulating powers are enfeebled, and depletion cannot be resorted to with advantage.

A striking and important feature in these two diseases, is the inefficiency of remedial means, än evil which is amply counterbalanced by the uniformly spontaneous abatement of the morbid action within a certain period. Observers are led to this humiliating conclusion after noticing precisely the same results from the administration of an infinite variety of medicines, and also from allowing the patient to remain quiet without the use of remedial means. Although this remark forcibly applies to the active stages of infuenza and cholera, it cannot be extended to the sequelæ of those diseases, where the judicious application of remedies is of the highest service.

Thus far a comparison and contrast between these mysterious affections may be sustained; to proceed further would be to enter a labyrinth which would obscure rather than elucidate the subject.

March, 1837.

ERo. 